\title{
Mixed reality serious games for post-stroke rehabilitation
}

\author{
Di Loreto Ines, Gouaich Abdelkader, Nadia Hocine \\ Lirmm - Université Montpellier 2 \\ \{diloreto,gouaich,hocine\}@lirmm.fr
}

\begin{abstract}
In this paper we propose a mixed reality system (MRS) and an adaptation module for rehabilitation of the upper limb after stroke through serious games. Aim of the system is to increase intensity and number of training session using the fun factor as driver. Although our system is targeting post stroke patient it is necessary to validate and test our approach with valid users at the first stage to provide patients with stable system to use. These studies and their results are described in this paper.
\end{abstract}

Keywords-Therapeutic Serious Games, post-stroke
rehabilitation

\section{INTRODUCTION}

Each year in the United States alone approximately 795.000 people are affected by stroke [6]. In France there are around 150.000 strokes each year [39], and similar trends are evident all around the industrialized world. Being the third cause of mortality and the first one of handicap in adults, we can say that stroke is one of the main health problems in our era [43]. In this paper we propose a mixed reality system (MRS) associated with a difficulty adaptation module for rehabilitation of the upper limb after stroke through serious games. Aim of the system is to increase intensity and number of training session using the fun factor as driver. Although our system is targeting post stroke patient it is necessary to validate and test our approach with valid users at the first stage to provide patients with stable system to use. These studies and their results are described in this paper.

In the following sections we will describe in more depth what a stroke is and what its consequences are, and why a 'virtual' approach could be useful as rehabilitation strategy. These sections are followed by a description of the proposed system and two experiments targeting health people.

\section{DESCRIBING STROKE}

A Cerebral Vascular Accident (CVA) or simply 'stroke' is traditionally defined by the World Health Organization as "a neurological deficit of cerebrovascular cause that persists beyond 24 hours or is interrupted by death within 24 hours" [43]. A stroke induces a loss of brain functions due to disturbance in blood supply to the brain, resulting in altered sensori-motor and/or cognitive functioning. The most striking motor impairment is the loss of motion control on the contralesional side. Because of this, stroke sufferers are often unable to independently perform day-to-day activities such as bathing, dressing and eating, inducing a loss of autonomy and a decrease in quality of life [43]. In addition, stroke is a highly heterogeneous syndrome. The specific abilities that will be affected by stroke depend on the location, type and size of the lesion. Each patient is then characterized by a specific combination of deficits [31]. Therefore, stroke rehabilitation programs are strongly personalized and not generic: they are adapted to a particular patient, to regain as much function as possible.

The most important gain of function takes place within the first six months after stroke. In most countries, due to high costs, only $6 / 8$ weeks of rehabilitation are executed under the direct supervision of an expert (i.e. in the hospital or rehabilitation centre). Following the hypothesis that the theories of motor learning can be applied on motor relearning most rehabilitation techniques are founded on principles of motor learning and skill acquisition established for the healthy nervous system. Acknowledged features are among others: (i) the motivation of the participant; (ii) the use of variable practice (i.e. practice a variety of related tasks); (iii) training with high intensity/many repetitions; (iv) and providing feedback [25],[26]. To experience significant recovery, stroke patients must then perform a substantial number of daily exercises. Unfortunately, typical rehabilitation sessions with therapists include a relatively small number of motions [24] due to the low duration of personal rehabilitation sessions. Home training can increase the amount of executed movements. However, while this is often prescribed, only $31 \%$ of the patients actually perform the recommended exercises [31]. This raises the problem of selecting a home-based rehabilitation technology that both can help and motivate patients to perform their therapeutic exercises to ameliorate their recovery after stroke.

Finally, as a side effect stroke patients can develop depression or aggressiveness due to the trauma of reduced capabilities. Depression and aggressiveness also imply that stroke survivors may find it difficult to focus on a therapy programme. While rehabilitation programmes attempt to stimulate the patient with a variety of rehabilitation exercises, stroke victims commonly report that traditional rehabilitation tasks can be boring due to their repetitive nature. We can then say that apart from repetition, also motivation is another important factor for the rehabilitation success.

\section{VIRTUAL REHABILITATION}

The challenge for post stroke rehabilitation is then to create exercises able to decrease the monotony of hundreds of 
repeated motions. In order to overcome this challenge, different kinds of 'non traditional' therapies have been proposed. For example, the possibility of using 'virtual' rehabilitation (hereafter VR) has been the subject of experiments by several authors (for example [35][21]). Although most of the studies on this topic are linked to the study of virtual reality environments recent works have focused on the use of videogames and consoles for rehabilitation (such as [5][14]).

To summarize results from these studies the interest of virtual rehabilitation is noticeable at three different levels.

\section{A. Increasing rehabilitation volume through motivation}

The first level is linked to the motivational aspect from the patient's point of view:

1) Personalization: VR technologies create an environment in which the intensity of feedback and training can be manipulated to create the most appropriate, individualized motor learning paradigm [21].

2) Interactivity: VR exercises can be made to be engaging so that the patient feels immersed in the virtual world. This is extremely important in terms of patient motivation [32], which in turn, is one of the key factors for recovery.

3) Feedback: Interactive feedback can contribute to motivation. By providing visual and auditory rewards such as displaying gratifying messages in real time, patients are motivated to exercise [7][16].

\section{B. Increasing rehabilitation volume through effectiveness}

The second level is linked to the effectiveness aspect:

4) Tracking: The evolution of the patient's performances can be easily stored, analysed and accessed by therapists [21][32]. The only purpose for the patient (but also for the therapist) is to recover the most of his/her body functionalities. It is therefore important to show the patient and the therapist patient's progresses during therapy.

\section{Increasing rehabilitation volume through 'telerehabilitation'}

The third one is linked to the portability and economical aspect (low cost of the devices):

5) Telerehabilitation: A VR system could be used outside rehabilitation centers, allowing for ubiquitous rehabilitation. However, before of this adoption it is necessary to demonstrate not only its medical efficiency but also to show that the system is economically sustainable. Cheaper personal equipment (pc-based for example) could eventually allow rehabilitation (i.e.) stations to be placed in locations other than the rehabilitation centre, such as patient's home.

\section{Limits of virtual rehabilitation}

On the other hand, Virtual Rehabilitation does raise significant challenges before its widespread adoption such as its clinical acceptance (which relies on proved medical efficacy); therapist's attitude towards the technology (e.g. the therapist fears that technology could replace therapists and the like); patient's attitude towards the technology (e.g. the patient may not consider a game to be 'real' rehabilitation); Ethical and regulation challenges linked to the kind of technology used.

In the rest of this paper we will describe: (i) several existing works on post stroke rehabilitation, (ii) an hand made system able to support serious games for post stroke rehabilitation, (ii) two experiments held and their results, (iii) some consideration on future improvements on the system.

\section{RELATED WORKS}

This section reviews existing VR approaches for post stroke rehabilitation and existing difficulty adaptation approaches for games.

\section{A. Virtual rehabilitation systems for stroke}

It's out of the scope of this paper to make an exhaustive presentation of all kinds of rehabilitation systems. For this reason only the two most used rehabilitation systems (robot based and virtual reality) are reviewed. A final section describes the mixed reality virtual rehabilitation approach, less used but very interesting from the portability aspect. In fact, the portability and the cost of the rehabilitation tool can influence its adoption for home based rehabilitation, and thus influence the amount of rehabilitation. For this reason in this section we will describe (i) the kind of system, (ii)it's invasiveness, (ii) it's portability (iv) it's cost.

Robot assisted rehabilitation: Robot based approach for rehabilitation has been used since the late 90 s to assist patients (see e.g. [28][30]). This kind of assisted rehabilitation shows interesting results as demonstrated by large clinical trials (see e.g. [28]). These results are explained by the amount of movements produced by the robots and executed by the patient. As an example of this kind of system we can cite the Manus Robot developed at the MIT [41]. In this system the patient is in front of the robot and her shoulder is strapped to a chair. The patient's impaired arm is strapped to a wrist carrier and attached to the manipulandum. A video screen is above the training table to create a virtual context for movements and provide visual feedbacks to the patients. This kind of approach (a robot that induces movements while the patient is looking at a screen) is a vastly used approach for game based rehabilitation. This kind of approach makes the eyes and hand acting on different places, which is a relevant effort for patients in firsts' rehabilitation phases. In addition, the robot is an invasive system requiring that the patient's arm and chest to be strapped. Finally, due to the unitary cost (about $10 \mathrm{KE}$ for the Manus robot) and setup complexity, therapies with the robot are intended to be performed in dedicated places.

Virtual reality: Latest works on virtual reality are based on motion capture (see e.g. [22][42]).. As an example we can cite the IREX VR [20]. The IREX VR is a projected video-capture device [23],[34]. Within this system participants are placed in front or a chroma key green scene and their image is merged, at realtime, with a virtual environment and projected on a monitor. A camera is used for tracking participant's movements and converting them to interaction events within the virtual environment. Since, the IREX requires a special chroma key green scene and a bright ambient light, rehabilitation sessions must be performed only in dedicated 
places. The patient sees her image on a monitor, which implies an additional cognitive effort to situate herself in the virtual world. No invasiveness is reported for IREX since the patient is free from any device. Finally, the IREX system cost is estimated between 5-10 KE.

The Sony EyeToy is a very different way to approach virtual rehabilitation through virtual reality. It's in fact an economic alternative to IREX for VR rehabilitation [33]. EyeToy is an off-the-shelf low cost gaming device that allows interaction with virtual objects displayed on a standard TV. By contrast to IREX, EyeToy requires neither a chroma key scene nor a special ambient light so it can be deployed anywhere opening opportunities for telerehabilitation. However, the therapist has to control both the patient and the monitor at the same time. The patient is free from any device which makes EyeToy non invasive. However, similarly to IREX, the patient has to make an additional cognitive effort to situate herself in the virtual environment and to coordinate her arms to interact with virtual objects.

Mixed Reality: Mixed Reality systems for post stroke rehabilitation are a relatively not explored realm [13]. Al Mahmud et al.,[1] have developed a social game (not conceived for rehabilitation purposes) using a tabletop augmented reality platform which could have interesting implication for post stroke rehabilitation. The Visual Interaction Platform (VIP) [3] allows interaction with physical objects that are tracked using infrared based system. A visual feedback is provided by projecting the display on the table. The patient interacts with her hand with objects on the table were the display is projected. This makes interaction very natural with both, eyes and hand, targeting the same place. Since no device is attached to the patient, the VIP is considered as not invasive. VIP device used in [1] is a home made prototype, so no information is available to estimate its cost. Besides, VIP is a quite large system so a dedicated place is required.

\section{B. Therapeutic Serious Game and Difficulty Adjustment}

As seen in Section III of this paper one of the advantages of this Virtual Rehabilitation is to use motivation to increase the rehabilitation volume. One of the ways to increase motivation is to personalize the rehabilitation activity adjusting the difficulty level basing on the patient's performances. For this reason hereafter we describe several existing works dealing with difficulty adjustment in games, in particular therapeutic games.

From a game design point of view, the game difficulty level can be designed in a static or a dynamic manner. The static approach consists in an objective determination of the difficulty level. In this case, the player can manually choose the difficulty level, or the game can propose to follow a series of difficulty levels. The dynamic approach, which has the greatest interest for rehabilitation purposes, focuses on adapting the game following the player progress, status and performances during the game experience [15][9]. In this context we can find for example works on dynamic difficulty adjustment (DDA) in entertainment games which consider difficulty as a heuristic function that depends on game metrics [19] or techniques based on the player's profiles [15]. In the therapy context, most of therapeutic games are based on the static determination of tasks difficulty by therapists [2]. For example, Heuser et al [17] suggest five therapeutic games exercises. Each exercise involves a set of difficulty levels following patient's recuperation. The difficulty level is designed in a static manner, thus, when the patient fails as the difficulty level is beyond his capabilities, the simulation stops the exercise.

An interesting example of dynamic difficulty balancing in therapy can be found in Chen Y. et al [8]. The authors propose a framework for media adaptation in task-oriented neuromotor rehabilitation based on biofeedbacks [18]. Scenery images and their clarity, sounds variety and music instruments are used to reflect the arm movement. The adaptation strategy depends on the choice of the musical instrument, tempo variation and task difficulty which is annotated by the therapist. The game uses a dynamic decision network to model and adjust the relationship between the media and the patient performance in real time.

Finally, Ma et al, [29] propose an in-between approach. The therapeutic game aims to improve functional arm capabilities, visual discrimination, and selective attention using three difficulty levels: Beginner, Intermediate and Expert. The system suggests a difficulty level to the patient according to his/her profile data (in particular the motricity index [11]). The adaptation technique is based on a specification matrix that determines the relationship between the patient profile and the difficulty level. The difficulty level is statically determined. Furthermore, the adaptation technique doesn't take into account the in-game player's performances and his/her conditions or situations such as his/her tiredness, motivation and engagement.

\section{PROPOSITION}

In section III (Virtual Rehabilitation) we stated that (i) motivation, (ii) effectiveness, and (iii) portability are factors that could enhance the volume of rehabilitation. In this section we will describe the approach we used and the systems we developed in order to address such elements.

\section{A. Portability and economical aspects: a mixed reality system}

In this section we describe the mixed reality system (MRS) conceived for post stroke rehabilitation purposes. In general a mixed reality environment aims to merge real and virtual worlds to create a new context of interaction where both physical and digital objects co-exist and interact consistently in real time. The mixed reality system we conceived and developed for this study is composed of three main subsystems: (i) the gaming subsystem responsible for managing the game application; (ii) the motion capture subsystem responsible for tracking patient's movement; and (iii) the display subsystem responsible for displaying the virtual game environment in a physical environment.

The gaming subsystem follows a client-server pattern: the mechanics of the game are implemented at the server level. The game server translates this input into game events that are used to compute the next state of the game according to the game mechanics. Once the new state of the game has been computed, update events are sent to the game client(s) to update their local 
copies of game objects, which in turn update the graphical environment. The game client fulfils the following functions: (i) receiving patient's movement events from the motion capture system; (ii) forwarding these events to the game server; (iii) receiving events from the game server to update game object state; and finally (iv) generating the game's output in terms of 3D scenes and sounds.

The motion capture subsystem is responsible for tracking the patient's hand and to communicate these movements to the game client. The motion capture system is composed of three components:

1) An IR emitter: this is an active sensor built using a simple infrared (IR) emitter that is attached on the patient's hand using a Velcro strap. This basic device is very cheap (less than 1 euro) and convenient to use even with patients that suffer from spasticity.

2) An IR camera: To track the IR emitter a Nintendo Wiimote was used as an IR camera. The Nintendo Wiimote is an affordable device (about 30 euros) that has been used in many open source projects.

3) A processing software: the role of this component is to translate Wiimote events into mouse events so that the IR emitter and IR camera are considered as a standard pointing device from the operating system's point of view.

The display subsystem: Within a mixed reality context it is necessary to display game objects directly in the physical world. Thanks to a new generation of mini video projectors, namely pico projectors, it is possible to display the game on a flat surface almost anywhere. Within the context of this experiment, the pico projector is attached to a support to display the game onto a standard table. Pico projectors are affordable (less than 200 euros), lightweight (less that $300 \mathrm{~g}$ ), and easy to use by simply plugging a VGA cable into a computer. However, they are limited in terms of brightness (the one that was used in this experiment was less than 30 lumens). This constraint has to be taken into account when building the game's graphical environment by selecting colours and contrasts to compensate for this limitation.

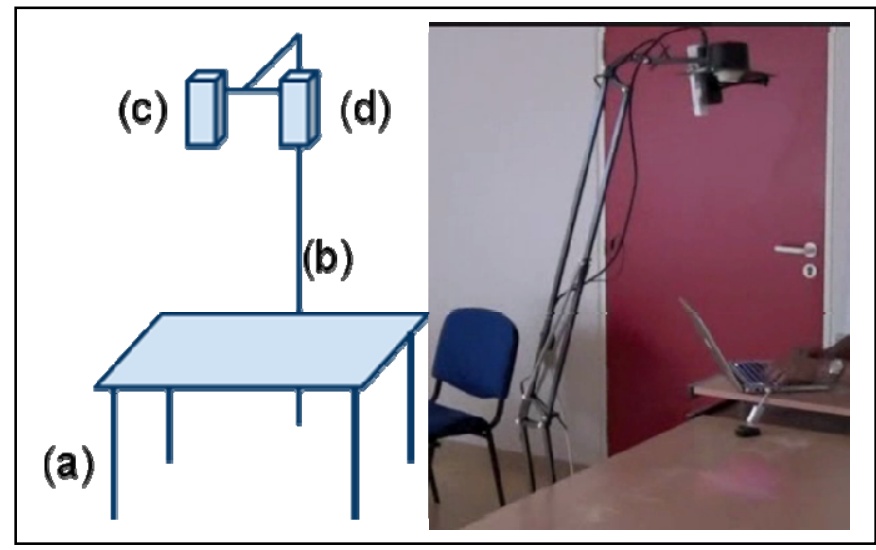

Figure 1. The overall system.

The overall system (Figure 1): The patient holds on his hand the IR emitter device and sits in front of the table (a) to play the exercise. The IR camera (c) and pico projector (d) are placed on the top of a support (b). A laptop computer is used to run the game client and to establish a Bluetooth connection with the WiiMote.

\section{B. The motivation aspect: adaptation and serious games}

The MR system described above is able to support different kinds of therapeutic games, conceived to train different types of movements. In particular, a module in the game server allows developers to integrate different types of adaptation for every game, offering a personalized gaming experience for each patient. Being each stroke different from another one a user centered approach is essential to contribute to the usability and acceptance of a therapeutic serious game.

In order to overcome these challenges we conceived a generic difficulty balancing technique dedicated to a family of therapeutic serious games for upper-limb rehabilitation. The proposed technique focuses on a real-time adaptation module based on a user-centered adaptation approach. The main objective of this technique is to improve the rehabilitation outcomes while maintaining the patient's engagement. To avoid monotony - and consequently patients' demotivation rehabilitation needs a variation of game themes and ambiances. However, although the theme and ambiance of the game can be changed, the therapeutic objectives and strategies have to be maintained, i.e. they have to be independent from the actual game. Consequently, two levels have been identified: (i) an abstract movement level and (ii) an actual game level. The abstract movement level considers the mechanics of patients' movement and their relationship to therapeutic objectives; the actual game level constructs a context and meaningfulness for the abstract level. For instance, at the abstract movement level the objective can be to reach a target point starting from a source point. This is translated differently in two games that we have developed: in one case, the objective is to scare crows eating the crop; in the second game, the goal is to catch an animated fish [12].

A general Difficulty Adjustment Framework: Once the therapeutic goal set (for example: increasing the range and smoothness of the impaired arm movement) a therapy session composed of a sequence of therapeutic games takes place. When interacting with the therapeutic games the patient generates a set of observable data that are collected and referred generically as performances. The adaptation module uses this data to adjust the player task difficulty. Our approach to motivation is inspired by the adequationist paradigm relating the motivation to the satisfaction of an individual [27]. In this approach, motivation is mainly considered as a regulation process. When reality - the outcome of a therapeutic game - is consistent with the patient's expectations - patient's perception of her/his own motor skills - the state is considered as a stable satisfaction state. The patient tries then to preserve this state. In the case of a minor disruption (constructive dissatisfaction) the patient may produce efforts to regulate her/his satisfaction state. In the case of important disruption however, the patient could fail in this efforts and entering in a demotivation situation. The difficulty adjustment module is asked to produce constructive dissatisfaction to keep the patient engaged in the 
therapy and then increase the rehabilitation volume (i.e. total time and number of exercises).

A motivation model: Several models of motivation exist and depend strongly on the application domain. To be applied in the context of post stroke therapeutic games, models have to take into account several factors such as: success and stimulation, feedback on activities, autonomy, self-esteem, meaningfulness and variety of tasks. Fort this first motivation model we have used the activation theory principles [36]. This theory states that stimulation is essential and a certain level of activation is required in order to make an individual sufficiently motivated to perform tasks and achieve goals. As a measure for the level of activation we have considered the ratio between the probability of successes and failures for a patient [36]. The cumulative activation score is simply a sum of all activation scores. This measure keeps a memory of all activation scores achieved by the patients. It provides a global trend of the motivation that is not affected by local variations. To represent local variations, we introduce also a derivative measure on activation scores: this measure indicates the direction of the current motivation score when compared by the previous one

An Abstract Model for Difficulty Adjustment: To conceive the difficulty adjustment module at the abstract level we introduced a structure called: matrix of action probabilities (MAP). This matrix represents the 2D plane of game and each place $A(i ; j)$ holds a probability that an action should be performed at this location by the patient. Having this information, the game level has to direct game actions towards places with highest probabilities. The advantage of this structure is (i) to offer a common interface to all games, (ii) to be personal to each patient. In fact, to construct the MAP it is necessary to collect information directly from the patient. Each place $\mathrm{D}(\mathrm{i} ; \mathrm{j})$ represents then the particular patient's probability of success and not a generic one. Finally, the difficulty level is expressed as a desired success probability (d) for the patient. This is interpreted as the willing of having the patient succeeding with the probability of $\mathrm{d} \%$ at least.

Process of Difficulty Adjustment: In our approach, the difficulty of a task is related with its probability of success. Higher success probability indicates low difficulty. Conversely, lower success probability indicates higher difficulty. The difficulty adjustment module takes one of three decisions depending of the profile and motivation of the patient: increase, decrease or maintain the current difficulty level of the training session. Three criteria are taken into account for adjusting the difficulty level. The first two criteria $\mathrm{S}^{+}$local ${ }^{(\mathrm{n})}$; $\mathrm{S}^{-}$local ${ }^{(\mathrm{n})}$ measure the local instability of the motivation in both direction (increasing and decreasing). The third criterion measures the overall trend of the motivation.

\section{EXPERIMENT THE SYSTEM}

This section will describe two experiment we held to test potential strength and weakness of the two above describe elements (the MR system and the adaptation module) for upper limb post stroke rehabilitation purposes.

\section{A. Therapists opinion and virtual rehabilitation systems}

A great majority of systems created for virtual rehabilitation purposes tests patient's acceptance and do not test in a systematic way therapists' opinions about the system (interesting exceptions are [4], [40]). On the contrary we are convinced that only a therapist can assess the rehabilitation value of a system. In fact, from a previous pilot study we held with 4 patients affected by stroke emerged clearly that the patient is not able to measure the impact of the system on her/his rehabilitation session/program. In that pilot study patients tested a game with a graphical tablet, a mouse and the mixed reality system described above. We can summarize results as follow. Firstly patients were not able to differentiate the difficulty of using the system from the difficulty of executing the movement, strongly impaired by the stroke. Secondly, the clinical eye of the therapist, who assisted the sessions, was able to observe for which patient the exercise was more difficult or when the patient had to put all efforts to move his hand (and thus was concentrating on his hand and not on the screen and the like). For this reason we decided that before testing the system with patients, it is really important to assess potential benefits with the therapists.

The Experiment: Aim of this experiment described was to compare the use of the in-house designed Mixed Reality System (MRS) with two alternative tools through an empirical investigation. The two alternative tools were a classical PC system, and the Wii system. In order to compare acceptance level of these systems (in Shackel terms, see [37]) we decided to assess perceived utility, usability, likeability (affective evaluation) of the three systems.

Participants: The mixed reality system described above has been tested on 17 members of the medical staff of a rehabilitation department of a French hospital. 3 occupational therapists, 9 physiotherapist, 3 students in physiotherapy and 2 general doctors participated in the study. Participants had an average of 15 years of experience.

The experiment: The experiment was composed of different sessions, each one testing a different 'form of potential therapy' (the Wii console with the Wiimote, computer and mouse, mixed reality with captor). The game on the computer and the one on the mixed reality system were identical. This was done in order to show that games and hardware system could be independent. The virtual rehabilitation game developed for this experiment was conceived to train upper limb 2D movements (i.e. movements on a plan without need to lift the arm). The gameplay stages a maize plantation and crows trying to steal maize. Aim of the game is to chase away all the crows; aim of the exercise is to train free movements. For each session therapists were let free to play with the system, but they were asked to keep in mind that they were playing the patient's role. Each session took 10 minutes. After the last session a questionnaire was conducted. Order of sessions was randomized.

The Questionnaire: The survey was conceptually divided into two parts. Results are expressed as a note on a four level scale, "4" being the best note and " 1 " being the lower one (Likert scale). The first part addressed the different hardware systems while the second one the games. In the first part, 
several questions addressed the usability of the different systems from the patient's point of view. Another group of questions addressed the perceived utility of the system for therapy. Finally, a last question compared usability of the three devices. In the second part of the survey, a set of questions addressed the different games utility for therapy, and the fun perception of each game. Each question asked also to motivate the rating.

Results and discussion: As said previously, the first set of questions was related to the system and did not concern the games.

The first question asked the therapists if they thought that patients will be able (from a physical point of view) to work with the three systems (considered as a whole). MR rated a means of $3.8( \pm 0.4)$; PC rated $3.1( \pm 0.9)$, the Wii system rated $2.9( \pm 0.9)$.Another question addressed the devices used with the three systems (the Wiimote, the mouse, the led captor). The usability for a patient of the IR Led rated a means of 3.3( \pm 1.2$)$, the mouse $2.2( \pm 1.2)$, the Wiimote $2.4( \pm 1.4)$.

To motivate these ratings the therapists wrote in synthesis that to be manipulated the Wiimote requires a set of motor skills that patients do not necessarily have regained. Most patients have also coordination problems. For those patients it will be difficult to support or release buttons on the controller in time. In addition, having to press a lever can generate spasticity or synkinesia (i.e. uncontrolled movements). While the subject of spasticity was raised by all the therapists (for both, the mouse and the Wiimote) several of them underlined that the Wiimote could be interesting in order to re-educate grasping.

On the other hand, in the therapists' words, the MR system is easy to understand and requires no cognitive effort by the patients. Finally, the sensor on the hand seems to me more suited for a larger number of patients.

Almost all the questionnaire in the end commented that the two systems (MR and Wii) could complement each other following the patient recovery. While the mixed reality system can be used in a more acute phase, the Wiimote becomes an interesting device for patients who have recovered better (remembering that not all the patients will attain this phase).

The therapists were then asked if they thought that working with the different systems could be useful for the patients. The MR rated 3.8 $( \pm 0.4)$, The PC rated 3.1 $( \pm 1.1)$ and the Wii rated $3.5( \pm 0.6)$. As for the usability questions, both, the Wii and the MR have their advantages. The mixed reality system allows for coordination and proprioception (i.e. the sense of the relative position of neighbouring parts of the body). In fact, the patient must try to move her/his limb in coordination with other parts of his/her body to reach a target. The MR allows also for better hand-eye coordination, as the hand moves within the game. In fact in both, the PC case and the Wii case, the patients have to look at another place (the screen) in order to perform the task. This kind of task requires an additional difficulty for patients in the first rehabilitation phase. Comments on PC usage are also related to limitations about screen dimension. On the other hand the Wii uses more planes in the space respect to our MR version which is more useful for joints. Finally, both allow the therapist to work with larger amplitudes than the traditional PC.

The second set of questions was related to games.

The first question addressed the usefulness of the ad-hoc game with respect to the commercial Wii game. As perceived utility the ad hoc game rated $3.6( \pm 0.6)$ while the Wii game rated $2.9( \pm 1.0)$. On the other hand the perceived fun of the ad hoc game was $3.0( \pm 1.0)$ while the Wii game rated $3.6( \pm 0.5)$. This is an indicator of two elements. While there is a need to create ad-hoc games for stroke rehabilitation, they need to be real games with the fun factor and not only simply exercises. Also in this case there are different needs in different parts of the rehabilitation. In fact, more complex games require patients with no cognitive or attention difficulties.

The final question comparing the utility for therapy of the three systems had the following results: MR rated 3.6 $( \pm 0.7)$, PC $2.9( \pm 1.1)$ and the Wii 3.2( \pm 0.7$)$. In this question one of the therapists stated clearly that the MR system is more suitable for patients in rehabilitation even if commercial games seem more fun.

To summarize, the Mixed Reality System has a higher potential to be useful for a huge number of patients. In fact, the most underlined problem with PC and Wii for rehabilitation the use of a hand held device - becomes one of potentiality of mixed reality. In addition, the mixed reality system uses objects from the natural environment as an interface to the computer. This intuitive way of interaction simplifies the communication between man and machine, especially for unpractised users. Finally, a MR system can be used by several persons because it is not restricted to one screen or one keyboard.

\section{B. Therapeutic Serious Game and Dificulty Adjustment}

Before experimenting with patients and disturb their classical rehabilitation program we conducted a pilot study with healthy players to experiment with the approach presented in section V. Nonetheless, the experimentation scheme with healthy players has to simulate difficulty conditions - based on movement coordination on a $2 \mathrm{D}$ plan- similar to the ones patients' could encounter during their rehabilitation program.

The Experiment: Aim of the experiment was comparing the difficulty level proposed by the adjustment algorithm to the difficulty level perceived by the player. The proposed game is structured as follow: given a flat 2D plan that can be lifted by the player and a starting point the goal is to stabilize a ball on a target point within a limited time. Depending on the location of the target the player find it more or less difficult to stabilize the ball on the target. The experiment follows an independentmeasures design with two independent groups. Group 1 used a naive strategy of difficulty adjustment that increases the difficulty level in case of success. Group 2 used our proposition for difficulty adjustment.

For both groups the experiment was conducted in a similar way. An initial assessment allows collecting prior data about the player equilibrium capabilities. The user profile was then constructed using the functional measurement deduced from this evaluation test. This initial assessment also produces the 
difficulty matrix for the first round of the game. In addition, at the end of each round player's performances are used to generate the task difficulty for the next round. Besides, at the end of each round, the player reports on his own perceived difficulty using the DP-15 scale [10].

Participants: Each group was composed by 4 persons with the following characteristics:

Group 1: 3Males 1Female $(25.75 \pm 1.7)$

Group 2: 1Males 3 Female $(25.75 \pm 3.3)$

Results: Results are reported as (Mean \pm Standard Deviation). All statistical analysis was performed using $\mathrm{R}$ (http://www.r-project.org) version 2.12.0.

Table 1 shows that Group 2 which was using the difficulty adjustment strategy reported less perceived difficulty $(\mathrm{M}=5.68$, $\mathrm{SD}=2.90)$ than group $1(\mathrm{M}=8.45, \mathrm{SD}=3.44)$ which was using a progressive difficulty adjustment strategy. This difference was significant, $\mathrm{t}(157.26)=-5.5152, \mathrm{p}=7.007 \mathrm{e}-08$

Table 2 shows that Group 2 which was using the difficulty adjustment strategy succeeded significantly more than Group 1 which was using progressive difficulty adjustment strategy, $\mathrm{x} 2(1 ; \mathrm{n}=162)=7.3907, \mathrm{p}=0.006$.

\section{TABLE I. PERCEIVED DIFFICULTY

\begin{tabular}{|l|l|}
\hline Group & Perceived difficulty \\
\hline 1 & $8.45 \pm 3.44$ \\
\hline 2 & $5.68 \pm 2.90$ \\
\hline
\end{tabular}

TABLE II. SUCCESS/FAILURE RATIO

\begin{tabular}{|l|l|l|}
\hline Group & Success & Failure \\
\hline 1 & 36 & 50 \\
\hline 2 & 49 & 27 \\
\hline
\end{tabular}

Discussion: The assessment and probabilistic reasoning to generate a task with a success ratio provides an acceptable approximation of users' difficulties on a 2D plan. In fact, task difficulty increase and decrease correspond not only to actual success and failure in the game, but also to the perceived difficulty decrease and increase of the game as a whole. On the other hand, we have observed that users are very influenced by recent performances. For example, when evaluating some tasks having the same difficulty level, some users have reported less perceived difficulty simply because they succeeded in previous games (so they were in a "positive" mood). The motivationbased difficulty adjustment is then important to avoid letting the user in an unbroken situation of failure so that the user can be again in a situation of success (and the other way round). This highlights the dynamic and temporal nature of motivation and frustration when playing games.

\section{GENERAL DISCUSSION}

Because it is the goal of therapists to guide the stroke patients through their rehabilitation we are convinced that the therapists are the most suited to understand the potential utility of a tool for rehabilitation. For this reason a study has been conducted to understand the potential and benefits of a mixed reality system we developed. In synthesis results shown that the MRS is seen as useful for a huger number of patients, in particular the ones with more acute diseases. As described in Section IV the MRS is a low cost system and can be easily moved. In addition, to experiment with the approach presented in section $\mathrm{V}$ we have conducted a pilot study with healthy players. The experimentation scheme simulates difficulty conditions - based on movement coordination on a 2D plan- similar to the ones patients' could encounter during their rehabilitation program. Results show that a motivationbased difficulty adjustment then important to avoid letting the user in an unbroken situation of failure so that the user can be again in a situation of success (and the other way round).

If we cross these results with the ones coming from the mixed reality experiment we can say that the system has the potential to be used with therapeutic serious games in post stroke rehabilitation not only in hospitals but also for home based rehabilitation. We believe in fact, that the most innovative and interesting part of the presented system is the mix of adaptive aspects combined with the ease of use in different environments of the same tool.

\section{CONCLUSIONS AND FUTURE WORKS}

In this paper we propose a mixed reality system (MRS) and an adaptation module for rehabilitation of the upper limb after stroke through serious games. Aim of the system is to increase intensity and number of training session using the fun factor as driver. Although our system is targeting post stroke patient it was necessary to validate and test our approach with valid users at the first stage to provide patients with stable system to use. For this reason, before testing the system with patients, we carried several studies with therapists and health people in order to understand the potential and the system benefits for rehabilitation purposes. Results from the experiments show that the system has the potential to be used with therapeutic serious games in post stroke rehabilitation not only in hospitals but also for home based rehabilitation.

However, while these experiments were conducted with healthy people, a test of the presented system with stroke patients has to be undertaken. The first experiment we programmed with patients will involve the use of the mixed reality system with patients and therapists each in they own role. The main idea is to compare results from this experiment with the previous one in order to understand the discrepancy between patients' attitudes and therapists' expectations towards the system. The second experiment will extend the study around the motivation-based model. We plan in fact to evaluate differences in term of learning skills between a classical approach and an approach with motivation-based difficulty adjustment. Finally, the last prototype will implement collaboration between several users in order to understand if a collaborative therapy is possible, and what is the role of motivation in this case.

\section{REFERENCES}

[1] Al Mahmud, A., Mubin, O., Shahid, S., Martens, J.-B., 2008. Designing and evaluating the tabletop game experience for senior citizens. Proceedings of the 5th Nordic conference on Human-computer interaction building bridges - NordiCHI '08, 403.

[2] Alankus, G., Lazar, A., May, M., Kelleher, C.: Towards customizable games for stroke rehabilitation, In Proceedings of the 28th international 
conference on Human factors in computing systems, ACM, 2113-2122 (2010)

[3] Aliakseyeu, D., Martens, J., Subramanian, S., Vroubel, M., Wesselink, W., 2001. Visual Interaction Platform. Interact 2001, 232-239.

[4] Annema J., Verstraete M., Abeele V., Desmet S, and Geerts D.: Videogames in therapy: a therapist's perspective. In: Proceedings of the 3rd International Conference on Fun and Games (Fun and Games '10). ACM, New York, NY, USA, pp. 94-98 (2010)

[5] Burke, J. W., McNeill, M. D. J., Charles, D. K., Morrow, P. J., Crosbie, J. H., McDonough, S. M.: Optimising engagement for stroke rehabilitation using serious games. In: The Visual Computer vol 25 (12), pp. 1085- 1099 (Aug. 2009)

[6] Center for Disease Control and Prevention: Stroke facts http://www.cdc.gov/Stroke/index.htm

[7] Chen, Y., Huang, H., Xu, W., Wallis, R. I., Sundaram, H., Rikakis, T., Ingalls, T., Olson, L., He, J.: The design of a real-time, multimodal biofeedback system for stroke patient rehabilitation. In: Proceedings of the 14th annual ACM international conference on Multimedia MULTIMEDIA'06, 763 (2006).

[8] Chen, Y., Xu, W., Sundaram, H., Rikakis, T., Liu, S.M.: A dynamic decision network framework for online media adaptation in stroke rehabilitation, ACM Transactions on Multimedia Computing, Communications, and Applications (TOMCCAP), ACM, $1\{38$ (2008)

[9] Conati C., Manske M.: Evaluating Adaptive Feedback in an Educational Computer Game, Proceedings of the 9th International Conference on Intelligent Virtual Agents, Amsterdam, The Netherlands (2009)

[10] Deligneieres, D.: La perception de la difficulté dans les taches motrices, HDR, Université de Montpellier 1,(1996)

[11] Demeurisse, G., Demol, O., Robaya, E.: Motor evaluation in vascular hemiplegia, European Neurology 19, 382-389 (1980)

[12] Di Loreto, I., Gouaich, A.: Mixed Reality Serious Games: The Therapist Perspective, Arxiv preprint arXiv:1011.1560 (2010)

[13] Edmans JA, Gladman JR, Walker M, Sunderland A, Porter A, Fraser DS: Mixed reality environments in stroke rehabilitation: development as rehabilitation tools. 5th International Conference of Disability, Virtual Reality \& Assoc Tech (ICDVRAT): 2004: Oxford, UK.

[14] Flynn, Sheryl, Palma, Phyllis, Bender, Anneke: Feasibility of Using the Sony PlayStation 2 Gaming Platform for an Individual Poststroke: A Case Report. In: Journal of Neurologic Physical Therapy Vol. 31 (4), pp. 180-189 (Dec. 2007)

[15] Georgios N., Yannakakis, John Hallam: Real-time Adaptation of Augmented-Reality Games for Optimizing Player Satisfaction, IEEE (2008)

[16] Goude, D., Bjork, S., Rydmark, M.: Game design in virtual reality systems for stroke rehabilitation. Studies in health technology and informatics Vol. 125 (4), pp. 146-8,(Jan. 2007).

[17] Heuser, A., Kourtev, H., Winter, S., Fensterheim, D., Burdea, G., Hentz, V., For-ducey, P.: Tele-Rehabilitation using the Rutgers Master II glove following Carpal Tunnel Release surgery, In 2006 International Workshop on Virtual Rehabilitation, IEEE publisher, 88-93 (2006)

[18] Huang, H., Chen, Y., Xu, W., Sundaram, H., Olson, L., Ingalls, T., Rikakis, T., He, J.: Novel design of interactive multimodal biofeedback system for neurorehabilitation Engineering in Medicine and Biology Society, EMBS'06. 28th Annual International Conference of the IEEE, $4925\{4928$ (2006)

[19] Hunicke R., Chapman V.: AI for Dynamic Difficulty Adjustment in Games, Challenges in Game Arti_cial Intelligence AAAIWorkshop, AAAI Press, San Jose, $91\{96$ (2004)

[20] IREX, 2003. IREX. URL http://www.irexonline.com

[21] Jack, D., Boian, R., Merians, A., Tremaine, M., Burdea, G., Adamovich, S., Recce, M., Poizner, H.: Virtual reality-enhanced stroke rehabilitation. In: IEEE transactions on neural systems and rehabilitation engineering : a publication of the IEEE Engineering in Medicine and Biology Society Vol. 9 (3), pp.308-318 (Sep. 2001).

[22] P. Keyani, G. Hsieh, B. Mutlu, M. Easterday, and J. Forlizzi, "DanceAlong: supporting positive social exchange and exercise for the elderly through dance," in CHI '05: CHI '05 extended abstracts on
Human factors in computing systems. New Press, 2005, pp. 1541-1544. [Online].

[23] Kizony, R., Raz, L., Katz, N., 2005. Video-capture virtual reality system for patients with paraplegic spinal cord injury. Journal of Rehabilitation Research and Development 42 (5), 595-607.

[24] Lang, C.E., M. J., Gnip, C. Counting repetitions: an observational study of outpatient therapy for people with hemiparesis post-stroke. In: Journal Neuro. Phys. Therapy Vol. 1 (31), pp. 3-10 (2007)

[25] Langhorne, P., Coupar, F., Pollock, A.: Motor recovery after stroke: a systematic review. In: The Lancet Neurology Vol. 8 (8), pp. 741-754 (Aug. 2009).

[26] Levin, M. F., Sveistrup, H., Subramanian, S. K.: Feedback and virtual environments for motor learning and rehabilitation, Schedae, 1, 19-36 (2010)

[27] Levy-Leboyer, C.: Re-motiver au travail. Developper l'implication de ses collaborateurs Paris: edition d'organisation (2007)

[28] Lo, A. C., Guarino, P. D., G.Richards, L., Haselkorn, J. K., andDaniel G. Federman, G. F.W., Ringer, R. J., Wagner, T. H., Krebs, H. I., Volpe, B. T., Bever, C. T., Bravata, P., 2010. Robot-Assisted Therapy for LongTerm Upper-Limb Impairment after Stroke. New England Journal of Medicine 362 (19), 1772-1783.

[29] Ma M., Charles D., McDonough S., Crosbie J., Oliver L., McGoldrick C.: Adaptive virtual reality games for rehabilitation of motor disorders, Universal Access in Human-Computer Interaction, Ambient Interaction (2007)

[30] Micera, S., Carrozza, M. C., Guglielmelli, E., Cappiello, G., Zaccone, F., Freschi, C., Colombo, R., Mazzone, a., Delconte, C., Pisano, F., Minuco, G., Dario, P., 2005. A Simple Robotic System for Neurorehabilitation. Autonomous Robots 19 (3), 271-284.

[31] National Stroke Association, 2010.National Stroke Association. Effects of Stroke

(2010), http://www.stroke.org/site/PageServer?pagename=EFFECT.

[32] Popescu, V. G., Burdea, G. C., Girone, M.and Bouzit, M., Hentz, V. R.: Orthopedic Telerehabilitation with Virtual Force Feedback. In: IEEE Transactions on Information Technology in Biomedicine Vol. 4 (1), pp. 45-51 (2000).

[33] Rand, D., Kizony, R., Weiss, P. L., 2004. Virtual reality rehabilitation for all : Vivid GX versus Sony PlayStation II EyeToy. Virtual Reality, 87-94.

[34] Reid, D., Hirji, T., 2004. The influence of a virtual reality leisure intervention program on the motivation of older adult stroke survivors: A pilot study. Physical \& Occupational Therapy in Geriatrics 21 (4), 119.

[35] Rizzo, K.: A swot analysis of the field of virtual reality rehabilitation and therapy. In: Presence: Teleoperators and Virtual Environments Vol. 14 (2), pp. 119-146 (2005)

[36] Scott, W. E.: Activation theory and task design. Organizational Behavior and Human Performance, 1, 3\{30 (1966)

[37] Shackel, B.: Usability - Context, framework, definition, design and evaluation. In: Shackel, B., Richardson, S. J. (Eds.), Human Factors for Informatics Usability. Cambridge University Press, pp. 21-31 (1991)

[38] Shaughnessy, M., R. B., Macko, R.: Testing a model of post-stroke exercise behavior. In: Rehabilitation Nursing: The Official Journal of the Assoc. of Rehabil. Nurses Vol. 1 (31), pp. 15-21(2006)

[39] Societe Francaise Neuro-Vasculaire, bilan 2010, http://sfnvfrance. com/.

[40] Tam, S.: An Environment for Stroke Therapy Game Authoring. PhD Thesis. Department of Computer Science \& Engineering - Washington University in St. Louis (2010)

[41] Volpe, B., Krebs, H., Hogan, N., 2001. Is robot-aided sensorimotor training in stroke rehabilitation a realistic option? Current opinion in neurology 14 (6), 745.

[42] P. L. Weiss, D. Rand, N. Katz, and R. Kizony, "Video capture virtual reality as a flexible and effective rehabilitation tool." Journal of neuroengineering and rehabilitation, vol. 1, no. 1, pp. 12+, Dec. 2004. [Online].

[43] World Health Organisation, http://www.who.int/en/ 